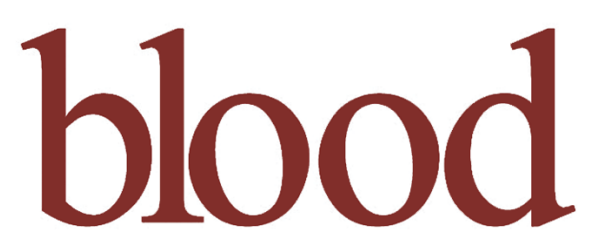

2012 119: 1561-1565

Prepublished online December 16, 2011;

doi:10.1182/blood-2011-11-391961

\title{
Thigh-length versus below-knee compression elastic stockings for prevention of the postthrombotic syndrome in patients with proximal-venous thrombosis: a randomized trial
}

Paolo Prandoni, Franco Noventa, Roberto Quintavalla, Carlo Bova, Benilde Cosmi, Sergio Siragusa, Eugenio Bucherini, Francesco Astorri, Stefano Cuppini, Fabio Dalla Valle, Anthonie W. A. Lensing, Martin H. Prins and Sabina Villalta

Updated information and services can be found at:

http://bloodjournal.hematologylibrary.org/content/119/6/1561.full.html

Articles on similar topics can be found in the following Blood collections

Clinical Trials and Observations (3461 articles)

Free Research Articles (1368 articles)

Thrombosis and Hemostasis (431 articles)

Information about reproducing this article in parts or in its entirety may be found online at:

http://bloodjournal.hematologylibrary.org/site/misc/rights.xhtml\#repub_requests

Information about ordering reprints may be found online at:

http://bloodjournal.hematologylibrary.org/site/misc/rights.xhtml\#reprints

Information about subscriptions and ASH membership may be found online at:

http://bloodjournal.hematologylibrary.org/site/subscriptions/index.xhtml

Blood (print ISSN 0006-4971, online ISSN 1528-0020), is published weekly by the American Society of Hematology, 2021 L St, NW, Suite 900,

Washington DC 20036.

Copyright 2011 by The American Society of Hematology; all rights reserved.

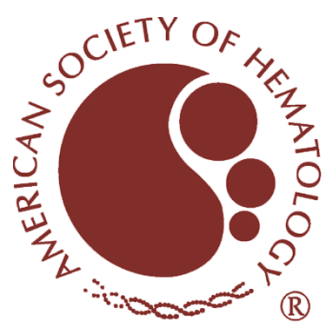




\title{
Thigh-length versus below-knee compression elastic stockings for prevention of the postthrombotic syndrome in patients with proximal-venous thrombosis: a randomized trial
}

\author{
Paolo Prandoni, ${ }^{1}$ Franco Noventa, ${ }^{2}$ Roberto Quintavalla, ${ }^{3}$ Carlo Bova, ${ }^{4}$ Benilde Cosmi, ${ }^{5}$ Sergio Siragusa, ${ }^{6}$ \\ Eugenio Bucherini, ${ }^{7}$ Francesco Astorri, ${ }^{8}$ Stefano Cuppini, ${ }^{9}$ Fabio Dalla Valle, ${ }^{1}$ Anthonie W. A. Lensing, ${ }^{10}$ Martin H. Prins, ${ }^{11}$ \\ and Sabina Villalta, ${ }^{12}$ on behalf of the Canano Investigators \\ ${ }^{1}$ Department of Cardiovascular and Thoracic Sciences, Clinica Medica 2 and ${ }^{2}$ Department of Clinical and Experimental Medicine, Group of Clinical \\ Epidemiology, University of Padua, Padua, Italy; ${ }^{3}$ Department of Internal Medicine and Angiology, University Hospital of Parma, Parma, Italy; ${ }^{4}$ Department of \\ Internal Medicine, Civic Hospital of Cosenza, Cosenza, Italy; ${ }^{5}$ Department of Angiology and Blood Coagulation M. Golinelli, S. Orsola-Malpighi University \\ Hospital of Bologna, Bologna, Italy; ${ }^{6}$ Department of Internal Medicine, Hematology and Transplantation Unit, University of Palermo, Palermo, Italy; ${ }^{7}$ Service of \\ Vascular Medicine and Angiology, Civic Hospital of Faenza, Faenza, Italy; ${ }^{8}$ Division of Internal Medicine, Civic Hospital of Fermo, Fermo, Italy; ${ }^{9}$ Division of \\ Internal Medicine, Civic Hospital of Rovigo, Rovigo, Italy; ${ }^{10} \mathrm{Centre}$ for Vascular Medicine, Academic Medical Centre, University of Amsterdam, Amsterdam, \\ The Netherlands; ${ }^{11}$ Department of Clinical Epidemiology and Technology Assessment, University of Maastricht, Maastricht, The Netherlands; and \\ ${ }^{12}$ Department of Internal Medicine, University Hospital of Treviso, Treviso, Italy
}

\begin{abstract}
Although below-knee compression elastic stockings (CES) are effective for the prevention of the postthrombotic syndrome (PTS), a substantial number of patients with deep venous thrombosis still develop PTS. In the present openlabel, randomized clinical trial, we compared thigh-length with below-knee CES for the prevention of PTS. A total of 267 patients with the first episode of proximal deep venous thrombosis were randomized to wear either thigh-length
\end{abstract}

or below-knee CES for 2 years. After 3 , $6,12,18,24$, and 36 months, they were assessed for PTS manifestations according to the Villalta scale. PTS developed in $44(32.6 \%)$ of the 135 patients randomized to thigh-length CES and in 47 (35.6\%) of the 132 allocated to below-knee CES, for an adjusted hazard ratio of 0.93 (95\% confidence interval, 0.62-1.41). Severe PTS developed in 3 patients in each group. CES-related side effects developed in 55 (40.7\%) of the 135 patients allo- cated to thigh-length CES and in 36 (27.3\%) of those randomized to the below-knee group $(P=.017)$, and led to premature discontinuation of their use in $29(21.5 \%)$ and $18(13.6 \%)$ patients, respectively. We conclude that thigh-length CES do not offer a better protection against PTS than below-knee CES and are less well tolerated. This study is registered as Clinical Trial number NCT00426075. (Blood. 2012; 119(6):1561-1565)

\section{Introduction}

Compression elastic stockings (CES) have been shown to offer substantial protection against the development of the postthrombotic syndrome (PTS) in patients with proximal deep venous thrombosis (DVT). ${ }^{1}$ Although the precise mechanism by which stockings reduce the risk of PTS is unknown, they are likely to counterbalance the effects of venous hypertension resulting from persistent venous obstruction and/or valve damage and to assist the muscle pump function..$^{2-5}$ The latest international guidelines recommend the use of CES in all patients with proximal DVT for at least 2 years. ${ }^{6,7}$

In 2 randomized studies in patients with proximal DVT, the use of below-knee CES for at least 2 years was associated with an approximately $50 \%$ reduction in the incidence of PTS compared with patients without CES. ${ }^{8,9}$ However, despite this large reduction, $25 \%$ of patients still develop postthrombotic manifestations, which are severe in $2 \%-5 \%$ of cases.

In clinical practice, many physicians recommend the use of thigh-length CES over below-knee CES, especially in patients with extensive proximal DVT, because of a perceived higher degree of protection against the development of PTS. ${ }^{4}$ To assess the potential benefit of thigh-length over below-knee CES for the prevention of PTS, we performed a multicenter randomized study in patients with a first episode of proximal DVT. In both study arms, patients were encouraged to use the CES for 2 years. The Villalta scale was used for the classification of PTS. ${ }^{10}$

\section{Methods}

\section{Study design and outcomes}

This randomized clinical trial compared the efficacy of thigh-length CES with that of below-knee CES used for 2 years for the prevention of PTS in patients with a first episode of proximal-vein thrombosis. The primary analysis concerned the 3-year cumulative incidence of PTS. In addition, compliance to the assigned CES and their tolerability was assessed.

\section{Patients}

Patients referred to 8 Italian university or hospital centers with symptomatic proximal DVT, as confirmed by compression ultrasonography, between October 2005 and September 2007 were potentially eligible for the study.
Submitted November 15, 2011; accepted December 13, 2011. Prepublished online as Blood First Edition paper, December 16, 2011; DOI 10.1182/blood2011-11-391961.

The publication costs of this article were defrayed in part by page charge payment. Therefore, and solely to indicate this fact, this article is hereby marked "advertisement" in accordance with 18 USC section 1734. 
Patients were excluded if they had recurrent ipsilateral DVT, preexisting leg ulcers or signs of chronic venous insufficiency, bilateral thrombosis, a short life expectancy, or contraindication for the use of CES (eg, advanced-stage peripheral arterial insufficiency or allergy to stockings).

Patients who passed the screen of inclusion and exclusion criteria were asked to participate in the study after receiving detailed written information about study hypotheses and procedures. Study patients had to give written informed consent to participate in the study in accordance with the Declaration of Helsinki. The study protocol was approved by the Institutional Review Board of each participating center.

\section{Anticoagulant treatment}

Patients were treated with low-molecular-weight heparin, overlapping with and followed by at least 3 months of vitamin $\mathrm{K}$ antagonist therapy (international normalized ratio [INR], 2.0-3.0), except for selected patients with active cancer or pregnancy, in which a low-molecular-weight heparin monotherapy was used.

The duration of anticoagulant treatment followed international guidelines with individual adaptations based on patient preferences and risk profile. In general, patients with transient risk factors were scheduled to receive up to 3 months of oral anticoagulant therapy, whereas patients with unprovoked thrombosis received at least 6 months of treatment and those with permanent risk factors such as active cancer were treated for the entire study period. The individual quality of oral anticoagulation was considered satisfactory if the INR was within or above the therapeutic range in more than $70 \%$ of determinations.

\section{Study procedures}

At baseline, a clinical history was taken detailing the presence of risk factors for venous thrombosis (ie, active cancer, recent $[<3$ months $]$ trauma or surgery, prolonged $[>7$ days] immobilization from medical illnesses, pregnancy or puerperium, or ongoing hormonal therapy). The search for thrombophilic abnormalities was left to the discretion of attending physicians

At the time of hospital discharge, usually 4-5 days after admission, patients were randomly allocated to wear either a thigh-length or a below-knee graded CES of the affected leg after wearing elastic bandages for the first few days at the discretion of attending physicians. Randomization was according to a computer-generated list that was accessible only to a trial nurse who informed study physicians on treatment allocation after patients had provided informed consent. Randomization was stratified by center, and permuted blocks of various sizes were used.

CES were supplied by Sigvaris AG. The stockings produced a pressure of $30-40 \mathrm{mmHg}$ at the ankle, were flat knitted, and composed of a combination of cotton, latex, and rubber/polyamide. Both thigh-length and below-knee CES were available in 3 sizes. Patients received 2 stockings, which were replaced by identical garments every 6 months. The CES had to be used during the day for a period of 2 years.

Patients were discharged with a letter for their family physician indicating that they had accepted to participate in a randomized study on CES. Both patients and their family physicians were given a card with the telephone numbers of the thrombosis clinic.

\section{Follow-up and assessment of recurrent thromboembolism}

Patients were followed for up to 3 years. The study was stopped when the last recruited patient completed the 3-year study period.

Patients were asked to visit the study center at 3 and 6 months from the index event and then every 6 months. In the interval between scheduled visits, patients were asked to report to the study center if a deterioration of symptoms or signs occurred. Patients who could not attend follow-up examinations at the study center were visited at home.

Patients were asked to return to the center if they developed symptoms suggestive of recurrent VTE. The diagnosis of recurrent thromboembolic events was made as described previously. ${ }^{11}$ For all patients who died during follow-up, the date and cause of death were recorded.

\section{Assessment of PTS}

Patients were instructed not to wear their CES on the day of assessment and not to reveal their treatment allocation to the assessor. Each PTS assessment was done by study personnel aware of the side of the index venous thrombosis but unaware of the treatment allocation and the results of previous measurements. Only the side of the index venous thrombosis was considered for the development of PTS.

The presence and severity of postthrombotic signs and symptoms were assessed at predefined times $(3,6,12,24$, and 36 months after the acute episode) using the Villalta scale. ${ }^{10}$ Briefly, the presence of 5 leg symptoms (ie, pain, cramps, heaviness, pruritus, and paresthesia) and 6 objective signs (ie, pretibial edema, induration of the skin, hyperpigmentation, new venous ectasia, redness, and pain during calf compression) was scored. For each item, a score of 0 up to 3 was assigned using the contralateral unaffected leg as the denominator for all evaluations. The presence of a venous ulcer of the lower limb indicated severe PTS regardless of the sum of the remaining signs and symptoms. In the absence of a venous ulcer, patients were classified as having severe PTS if they had a score of 15 or more on 2 consecutive visits at least 3 months apart. A total score of 5-14 on 2 consecutive visits at least 3 months apart indicated mild PTS. All other patients, including those who had a score higher than 4 on a single examination, were interpreted as not having PTS.

An independent adjudication committee whose members were not involved in the clinical assessments and who were unaware of treatment allocation evaluated the scoring forms at the end of follow-up and classified the outcome status of the patients.

\section{Compliance, adverse effects, and co-interventions}

Patients were asked to document their use of the CES, the occurrence of side effects, and co-interventions. For this purpose, they were instructed to record every day in a booklet the duration they wore the assigned CES, the use of unpermitted CES, the occurrence of possible adverse effects (such as itching, erythema, or other forms of allergic reaction), and the use of analgesic or anti-inflammatory drugs (including aspirin). The booklets were to be brought to the study physicians on the day of visit. Compliance was defined as satisfactory if CES were reportedly used for at least $70 \%$ of the time during the day.

\section{Statistical analysis}

Based on the assumption that the overall 3-year rate of PTS would be approximately $30 \%$ in the below-knee group, 313 patients would be required in each group to give a power of 0.80 and a 2 -sided significance level of 0.05 for the detection of 33\% risk reduction (from 30\%-20\%) with the thigh-length CES.

An interim analysis was planned after inclusion of approximately 250 patients to review these assumptions. Based on the obtained findings (hazard ratio [HR], 0.93; 95\% confidence interval [95\% CI], 0.62-1.41), the assumed reduction in the rate of overall PTS with the use of full-length CES was deemed unlikely to be achieved. Indeed, a sample size of 3951 patients in each group would have been necessary to confer a statistically significant value to the observed trend in favor of the full-length CES. Therefore, continuation of the study was deemed futile and inclusion of patients was stopped.

The primary analysis was conducted on an intention-to-treat basis. Cumulative incidences of PTS were calculated using the Kaplan-Meier method. Patients were censored after the last visit, in case of loss to follow-up or death. HRs and $95 \%$ CIs for the effects of thigh-length versus below-knee CES were calculated using the Cox regression models adjusted for age, gender, clinical presentation of DVT (unprovoked or secondary), and extent of the index thrombotic episode (popliteal only or common femoral vein with or without the involvement of the popliteal vein).

The $\chi^{2}$ test was used for comparison of compliance and tolerability. $P<.05$ was regarded as statistically significant. All calculations were performed with SPSS Version 18.0 software. 
Table 1. Main characteristics of the study patients

\begin{tabular}{|c|c|c|}
\hline & $\begin{array}{l}\text { Thigh-length } \\
\text { CES }(n=135)\end{array}$ & $\begin{array}{l}\text { Below-knee } \\
\text { CES }(n=132)\end{array}$ \\
\hline Mean age, y (range) & $67(20-94)$ & $69(21-90)$ \\
\hline Male sex, n (\%) & $69(51.1)$ & $73(55.3)$ \\
\hline Obesity, BMI > 30, n (\%) & $26(19.2)$ & $28(21.2)$ \\
\hline Thrombophilia in those tested, $\mathrm{n}(\%)$ & $30 / 93(32.3)^{\star}$ & 25/89 (28.1)† \\
\hline \multicolumn{3}{|l|}{ Clinical presentation, $\mathrm{n}(\%)$} \\
\hline Unprovoked & $80(59.3)$ & $82(62.1)$ \\
\hline Secondary & $55(39.6)$ & $50(37.9)$ \\
\hline \multicolumn{3}{|l|}{ Risk factors, $\mathbf{n}(\%)$} \\
\hline Recent trauma or surgery & $22(16.3)$ & $16(12.1)$ \\
\hline Malignancy & $12(8.9)$ & $15(11.4)$ \\
\hline Medical diseases & $12(8.9)$ & $14(10.6)$ \\
\hline Hormonal treatment & $7(5.2)$ & $4(3.0)$ \\
\hline Pregnancy or puerperium & $2(1.5)$ & $1(0.8)$ \\
\hline \multicolumn{3}{|l|}{ Location of DVT, $n(\%)$} \\
\hline Popliteal only & $51(37.8)$ & $64(48.5)$ \\
\hline Common femoral (with or without popliteal) & $84(62.2)$ & $68(51.5)$ \\
\hline \multicolumn{3}{|l|}{ DVT treatment } \\
\hline LMWH/VKA, n (\%)‡ & $124(91.9)$ & $120(90.9)$ \\
\hline UFH/VKA, n (\%) & $11(8.1)$ & $12(9.1)$ \\
\hline VKA duration, $\mathrm{mo}($ mean $\pm \mathrm{SD})$ & $10.3 \pm 9.2$ & $9.6 \pm 8.6$ \\
\hline
\end{tabular}

LMWH indicates low-molecular-weight heparin; UFH, unfractionated heparin; and VKA, vitamin $\mathrm{K}$ antagonists.

${ }^{*} \mathrm{FVL}$ in 11 patients, PTM in 10, APL in 4, deficiency in AT in 2, deficiency in prot S in 1 , and combined abnormalities in 2 .

FFVL in 11 patients, PTM in 7, APL in 4, and combined abnormalities in 3.

fEleven patients with cancer in each group were treated with LMWH monotherapy.

\section{Results}

\section{Patients}

Between October 2005 and September 2007, 363 potentially eligible patients with acute proximal DVT were considered for inclusion. Of these, 89 were excluded because of poor life expectancy $(n=36)$, previous ipsilateral DVT $(n=22)$, preexisting leg ulcers or venous insufficiency $(n=13)$, current use of elastic stockings $(n=11)$, bilateral thrombosis $(n=4)$, or known skin reactions to CES $(n=3)$. Of the remaining 274 patients, $267(97 \%)$ agreed to participate and were randomized to the thigh-length $(n=135)$ or below-knee $(n=132)$ CES. The baseline characteristics of the patients in the 2 treatment arms were similar (Table 1). Figure 1 shows a flow diagram that maps inclusion and progress of study participants.

During the study period, 11 patients were lost to follow-up ( 8 in the thigh-length group and 3 in the below-knee group) and 31 died (14 in the thigh-length group and 17 in the below-knee group). In none of the patients was death attributed to pulmonary embolism. The average duration of follow-up was $32.5 \pm 8.3$ months in the thigh-length, and $32.6 \pm 8.2$ months in the below-knee group.

During the study period, 12 patients in each group $(8.9 \%$ in the thigh-length and $9.0 \%$ in the below-knee group) developed nonfatal recurrent VTE, with involvement of the initially affected leg in 6 and 9 patients, respectively.

\section{PTS}

PTS developed in 44 of the 135 (32.6\%) patients (severe in 3) assigned to thigh-length CES and in 47 of the 132 (35.6\%) patients allocated to below-knee CES (severe in 3), a difference of $3 \%$ (95\% CI, - 14.2-8.3). The cumulative incidence of PTS after
3 years was $33.9 \%$ (95\% CI, 25.7-42.1) in the thigh-length group and $36.7 \%$ (95 CI\%, 28.3-45.2) in the below-knee group (Figure 2). The HR for PTS in the thigh-length compared with the below-knee group was 0.92 (95\% CI, 0.61-1.38). After adjustment for baseline characteristics, the HR remained unchanged $(0.93 ; 95 \% \mathrm{CI}$, 0.62-1.41).

Among patients with DVT involving the popliteal vein alone, 19 of $51(37.3 \%)$ developed PTS in the thigh-length group and 23 of $64(35.9 \%)$ in the below-knee group (HR 1.01; 95\% CI, $0.55-1.85)$, whereas these incidences were 25 of 84 (29.8\%) and 24 of $68(35.3 \%)$, respectively, among patients with a more proximal location of DVT (HR 0.86; 95\% CI, 0.49-1.51).

\section{Adverse effects, compliance, and co-interventions}

CES-related side effects (ie, itching, erythema, or other forms of allergic reaction) developed in $55(40.7 \%)$ of the 135 patients allocated to the thigh-length CES and in 36 (27.3\%) of those randomized to the below-knee group $(P=.017)$, and led to premature discontinuation of their use in $29(21.5 \%)$ and $18(13.6 \%)$ patients, respectively $(P=.11)$.

Compliance was good in 90 of 135 patients (66.7\%) in the thigh-length CES group versus 109 of 132 patients $(82.6 \%)$ in the below-knee CES group $(P=.003)$. When the analysis was confined to patients who did not prematurely discontinue the use of CES, compliance was good in 79 of 106 patients $(74.5 \%)$ in the thigh-length group and in 97 of $114(85.1 \%)$ in the below-knee group $(P=.063)$. Among the 199 patients who were compliant with their CES, the adjusted HR for PTS was 1.1 (95\% CI, 0.69-1.76).

Analgesic and/or anti-inflammatory drugs were used for variable periods of time ranging from a few days to 3 weeks by 10 patients in the thigh-length group and by 7 in the below-knee group.

Of the patients allocated to the thigh-length group, oral anticoagulants were administered for up to 6 months in $88(65.2 \%)$ and for longer periods in the remaining 47 ; the corresponding figures in the below-knee group were 88 (66.7\%) and 44, respectively. The quality of oral anticoagulant treatment was similar in both groups; an INR within or above the targeted therapeutic range (INR, 2.0-3.0) on at least $70 \%$ of measurements was reached in $90(66.7 \%)$ patients in the thigh-length group and in $88(66.7 \%)$ patients in the below-knee group.

\section{Discussion}

Despite appropriate anticoagulant therapy, 25\%-50\% of patients develop long-term posthrombotic sequelae after an episode of DVT. ${ }^{12}$ Established PTS remains a significant cause of chronic illness, with considerable socioeconomic consequences for both patients and health care providers. ${ }^{13,14}$ Among factors that have been found to be associated with an increased risk of PTS are proximal location of the initial thrombosis, especially the iliofemoral location, the development of recurrent ipsilateral DVT, and the inadequacy of oral anticoagulant therapy. ${ }^{9,15,16}$ The incidence of PTS is reduced by the use of graduated compression stockings. ${ }^{1,8,9}$ However, uncertainty remains about the choice of the most suitable type of stocking, because in proper studies on the value of CES for the prevention of PTS, only below-knee stockings were used. ${ }^{8,9}$ Thigh-length CES are generally perceived as being more effective, especially in patients with the most proximal involvement of the venous system, ${ }^{4}$ but are more expensive and may not be as practical and tolerable as the below-knee CES. To assess the relative role of 


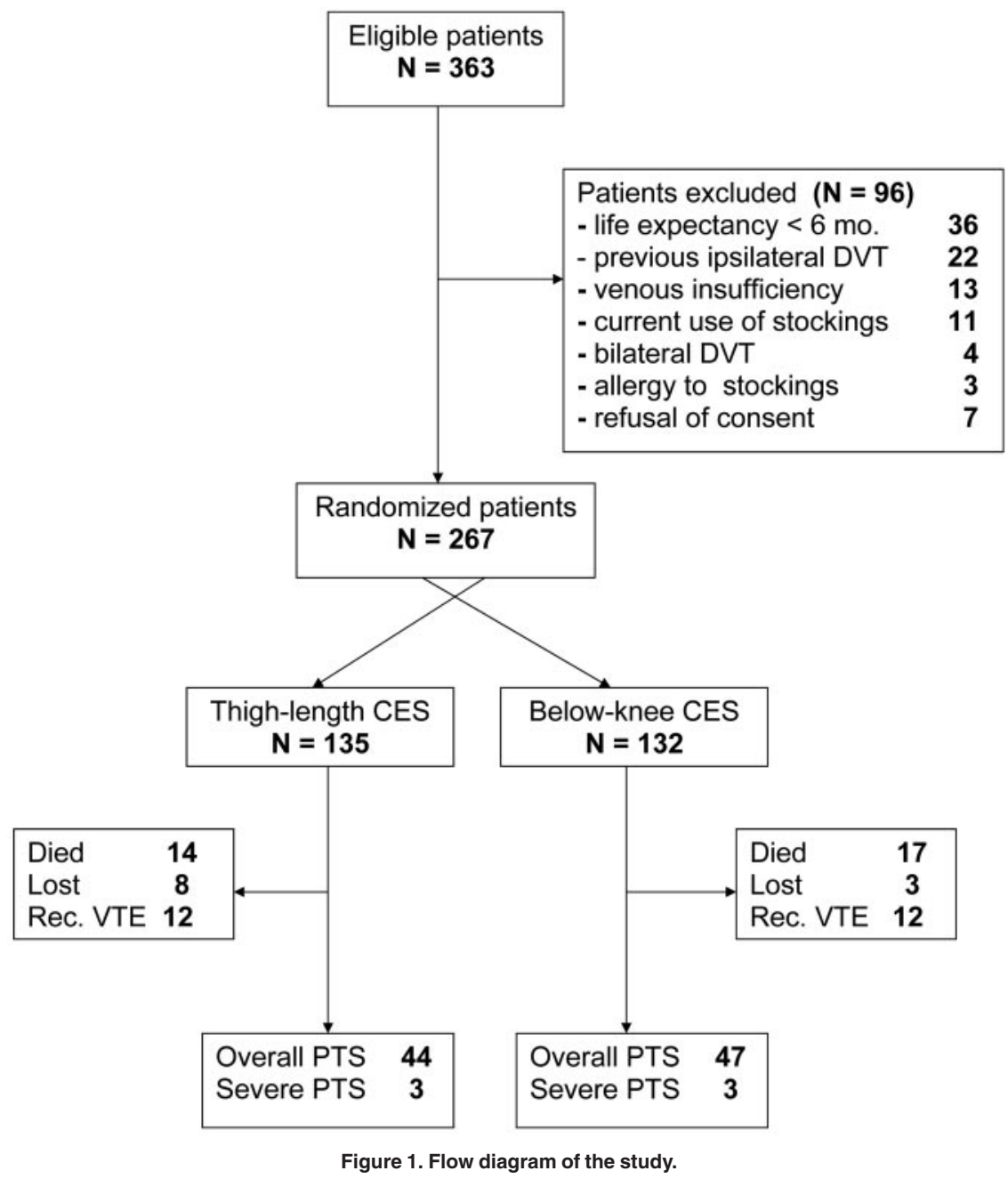

these 2 types of garments, we conducted a study to make a direct comparison.

We failed to show any advantage of the thigh-length over the below-knee CES. After completion of the 3-year follow-up, PTS developed in $32.6 \%$ of patients randomized to thigh-length and in $35.6 \%$ allocated to below-knee CES, for an adjusted HR of 0.93 (95\% CI, 0.62-1.41). Although the 95\% CIs are relative wide and potentially allow for a $30 \%-40 \%$ reduction in the incidence of PTS in patients wearing the thigh-length CES, such a reduction is unlikely, because the point estimates are close to unity. Even when the analysis was confined to patients compliant with their CES and those with the involvement of the common femoral vein, no benefit of thigh-length over below-knee CES was observed (HR, 1.1; 95\% CI, 0.69-1.76; and HR, 0.86; 95\% CI, 0.49-1.51, respectively).

The slight difference in the number of patients allocated to below-knee CES who developed PTS between the present study $(35 \%)$ and our previous study $(25 \%)^{9}$ most likely reflects the difference in patient age. Age was indeed found to be an important predictor of PTS development, $, 9,17$ and in the current investigation, patient age (mean, 67-69 years) was substantially higher than that (mean, 60-63 years) recorded in the previous study. Interestingly, in both studies, the rate of severe PTS was consistently low.

Not unexpectedly, the thigh-length CES were significantly less well tolerated. Indeed, a higher number of patients allocated to thigh-length CES developed CES-related side effects $(40.7 \%$ vs $27.3 \%$ ), which led to premature discontinuation in $21.5 \%$ and
$13.6 \%$, respectively. In addition, only $67 \%$ of the patients allocated to thigh-length CES used their garments for at least $70 \%$ of the time during the day, compared with $83 \%$ of patients allocated to the below-knee CES.

Some methodological issues require comment. Only patients with proximal DVT who were admitted to hospital departments were eligible for the current investigation. Therefore, the study conclusions cannot necessarily be generalized to those patients

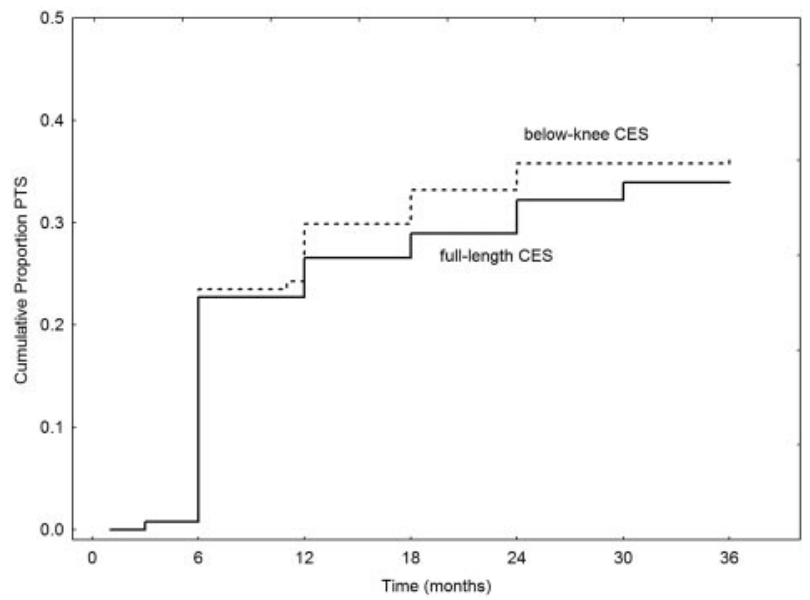

Figure 2. Cumulative incidence of PTS in the 2 study groups. 
who are managed entirely out of hospital. Because of the open design of the study, precautions were taken to avoid bias in the assessment of PTS. Therefore, an investigator who was not involved in the care of the patient and who was unaware of previous findings assessed the patient and completed the scale for PTS. For this purpose, patients were instructed not to wear their CES on the day of assessment and not to disclose information about treatment allocation to the assessor. Another physician independently collected information on protocol compliance at each follow-up visit. In addition, a blinded adjudication committee evaluated the scoring forms at the end of follow-up. Finally, the length of CES use (2 years) was decided in agreement with the latest American College of Chest Physicians guidelines, ${ }^{6}$ and the diagnosis of PTS was based on a validated scale with good correspondence to a patient's perception of quality of life and a high reproducibility. ${ }^{17}$

Below-knee CES should be regarded as the stockings of choice for the prevention of PTS in patients with proximal DVT. Indeed, they have similar effectiveness as thigh-length CES but with better tolerability and lower cost. The below-knee CES used in this study are currently marketed in Europe at the price of approximately 42 Euros compared with 75 Euros for the thighlength CES (ie, the below-knee CES are 56\% less expensive).

Although a considerable number of patients wearing belowknee CES still develop PTS, the rate of severe sequelae is acceptably low. Whether aggressive treatment of the initial thrombosis can further decrease PTS rate remains to be demonstrated, as does the assessment of the optimal duration of CES for its prevention.

\section{Authorship}

Contribution: P.P., A.W.A.L., M.H.P., and S.V. designed the study and wrote the manuscript; R.Q., C.B., B.C., S.S., E.B., F.A., S.C., and F.D.V. recruited the study participants; F.N. and M.H.P. analyzed the data; P.P. was responsible for administrative, technical, and logistical support; and all authors agreed on the final version of the manuscript.

Conflict-of-interest disclosure: The authors declare no competing financial interests.

Additional investigators of the Canano Study in order of the number of patients contributed were: V. Vedovetto, S. Barbar, E. Campello, M. Milan, and L. Filippi (Padova, Italy; $n=69$ ); A. Rocci (Parma, Italy; $\mathrm{n}=59$ ); P. Chiappetta, R. Pellegrini, E. Fiaschi, G. Vallone, E. D'Amico, and A. Noto (Cosenza, Italy; $\mathrm{n}=30$ ); C. Pili, E. Costantini, M. Gasperon, and G. Palareti (Bologna, Italy; $\mathrm{n}=28$ ); A. Malato and G. Saccullo (Palermo, Italy; $n=23$ ); F. Ventura and C. Brini (Faenza, Italy; $n=21$ ); G. Bitti (Fermo, Italy; $\mathrm{n}=20$ ); and M. Marzolo, E. Ramazzina, and S. Zamboni (Rovigo, Italy; $\mathrm{n}=17$ ).

Correspondence: Dr Paolo Prandoni, Department of Cardiothoracic and Vascular Sciences, Clinica Medica II, University of Padua, Via Giustiniani, 2, 35128 Padua, Italy; e-mail: paoloprandoni@tin.it.

\section{References}

1. Musani MH, Matta F, Yaekoub AY, Liang J, Hull RD, Stein PD. Venous compression for prevention of postthrombotic syndrome: A meta-analysis. Am J Med. 2010;123(8):735-740.

2. Pierson S, Pierson D, Swallow R, Johnson G Jr. Efficacy of graded elastic compression in the lower leg. JAMA. 1983;249(2):242-243.

3. Evers EJ, Wuppermann T. Effect of different compression therapies on the reflux in deep veins with a post-thrombotic syndrome. Vasa. 1999; 28(1):19-23.

4. Kahn SR, Elman E, Rodger MA, Wells PS. Use of elastic compression stockings after deep venous thrombosis: a comparison of practices and perceptions of thrombosis physicians and patients. J Thromb Haemost. 2003;1(3):500-506.

5. Prandoni P, Frulla M, Sartor D, Concolato A, Girolami A. Vein abnormalities and the postthrombotic syndrome. J Thromb Haemost. 2005; 3(2):401-402.

6. Kearon C, Kahn SR, Agnelli G, Goldhaber S, Raskob GE, Comerota AJ. Antithrombotic therapy for venous thromboembolic disease: American College of Chest Physicians Evidence-Based
Clinical Practice Guidelines (8th Ed). Chest. 2008;133(6 suppl):454S-545S.

7. Cardiovascular Disease Educational and Research Trust. Prevention and treatment of venous thromboembolism. International Consensus Statement (guidelines according to scientific evidence). Int Angiol. 2006;25(2):101-161.

8. Brandjes DP, Buller HR, Heijboer H, et al. Randomised trial of effect of compression stockings in patients with symptomatic proximal-vein thrombosis. Lancet. 1997;349(9054):759-762.

9. Prandoni $\mathrm{P}$, Lensing AWA, Prins $\mathrm{MH}$, et al. Belowknee elastic compression stockings to prevent the post-thrombotic syndrome. A randomized, controlled trial. Ann Intern Med. 2004;141(4):249-256.

10. Kahn SR, Partsch $H$, Vedantham S, Prandoni $P$, Kearon C. Definition of post-thrombotic syndrome of the leg for use in clinical investigations: a recommendation for standardization. J Thromb Haemost. 2009;7(5):879-883.

11. Prandoni P, Prins MH, Lensing AWA, et al. Residual thrombosis on ultrasonography to guide the duration of anticoagulation in patients with deep venous thrombosis: a randomized trial. Ann Intern Med. 2009;150(9):577-585.
12. Prandoni P, Kahn SR. Post-thrombotic syndrome: prevalence, prognostication and need for progress. Br J Haematol. 2009;145(3):286-295.

13. Bergqvist $D$, Jendteg $S$, Johansen $L$, Persson $U$, Odegaard K. Cost of long-term complications of deep venous thrombosis of the lower extremities: an analysis of a defined patient population in Sweden. Ann Intern Med. 1997;126(6):454457.

14. Kahn SR, Ginsberg JS. Relationship between deep venous thrombosis and the postthrombotic syndrome. Arch Intern Med. 2004;164(1):17-26.

15. Kahn SR, Shrier I, Julian JA, et al. Determinants and time course of the postthrombotic syndrome after acute deep venous thrombosis. Ann Intern Med. 2008;149(10):698-707.

16. van Dongen CJ, Prandoni P, Frulla M, Marchiori A, Prins $\mathrm{MH}$, Hutten BA. Relation between quality of anticoagulant treatment and the development of the postthrombotic syndrome. J Thromb Haemost. 2005; 3(5):939-942.

17. Kahn SR, Hirsch A, Shrier I. Effect of postthrombotic syndrome on health related quality of life after deep venous thrombosis. Arch Intern Med. 2002;162(10):1144-1148. 